\title{
The relationship between empathy and stress: a cross- sectional study among undergraduate medical students
}

\author{
James Wiguna Wahjudi', Ardi Findyartini ${ }^{2,3}$ and Fransiska Kaligis ${ }^{4,5}$ \\ ${ }^{1}$ Undergraduate Medical Program, ${ }^{2}$ Department of Medical Education, ${ }^{3}$ Medical Education Center, Indonesia \\ Medical Education and Research Institute (IMERI), and ${ }^{4}$ Department of Psychiatry, Faculty of Medicine, Universitas \\ Indonesia, and ${ }^{5}$ Department of Psychiatry, Dr. Cipto Mangunkusumo Hospital, Central Jakarta, Indonesia
}

Purpose: Empathy is critical for medical doctors, as it enables them to conduct good patient-centred care. Medical students are expected to learn this ability as part of their education and training.

Methods: Using a cross-sectional design, the present study was conducted to identify whether the empathy levels of medical students are affected by their stress levels. A translated version of the Perceived Stress Scale-10 was used to measure the students' stress levels, while the Jefferson Scale of Physician Empathy was used to measure their empathy levels.

Results: A total of 464 students from one medical school in Indonesia participated in the study. Stress levels among medical students peak in their first year of study and maintain a downward trend over the following years. The students' empathy levels increased during their first 3 years, declined significantly upon entering the first clinical year, and increased during the second clinical year. However, no correlations were found between stress level and empathy level.

Conclusion: These findings suggest that there may be other underlying factors that contribute to empathy decline among medical students upon entering their first clinical year. Further research should be conducted to identify these factors. The bounced-back of empathy level to a higher level in the second year highlights the importance of student adaptation in the clinical learning environment and the support system.

Key Words: Empathy, Stress, Undergraduate, Medical students

\section{Introduction}

Medical education has often been identified as a major source of stress for its students [1,2]. Compared to the general population, medical students have higher levels of psychological stress, which may have negative impacts on their performance and mental health [1]. Studies have also found that high stress levels can impair one of the most important abilities among physicians: clinical empathy [1,3,4]. However, it should be understood that stress, as a nonspecific response of the human body to external triggers, can be divided into two categories: "eustress," referring to stress which leads to better performance and productivity, and "distress," which causes anxiety, anger, and depression [5]. Both academic and non-academic factors can influence the stress levels of medical students. In the preclinical years, stressors are mostly related to the students' level of adaptation to new study methods, surroundings, and
Received: May 11, 2019 • Revised: July 3, 2019 • Accepted: July 22, 2019 Corresponding Author: Ardi Findyartini (https://orcid.org/0000-0002-9601-3994) Department of Medical Education, Faculty of Medicine, Universitas Indonesia, Salemba 6, Central Jakarta 10430, Indonesia

Tel: +62.213901814 Fax: +62.213901814 email: ardi.findyartini @ui.ac.id, findyartini@yahoo.com
Korean J Med Educ 2019 Sep; 31(3): 215-226.

https://doi.org/10.3946/kjme.2019.132

eISSN: 2005-7288

(C) The Korean Society of Medical Education. All rights reserved. This is an open-access article distributed under the terms of the Creative Commons Attribution Non-Commercial License (http:// creativecommons.org/licenses/by-nc/3.0/), which permits unrestricted non-commercial use, distribution, and reproduction in any medium, provided the original work is properly cited. 
academic performance. Clinical years can also be stressful for students, as they are faced with intense academic pressure, ethical dilemmas, and anxiety when encountering dying patients [1].

"Empathy" can be defined as the act of understanding another individual's emotional condition without $\mathrm{ex}^{-}$ periencing that condition itself, which involves both cognitive and emotional aspects [6]. "Clinical empathy" can be defined as a skill that allows medical doctors to understand their patients' feelings, conditions, and perspectives, communicating and acting on these understandings without adopting the patients' emotions $[3,7,8]$. "Clinical empathy" therefore allows medical doctors to "stand in the patient's shoe" without being involved with the patient's emotions and it is considered a core component of an effective physician $[3,7,8]$.

In the doctor-patient relationship, clinical empathy makes a significant contribution toward improving quality of care, communication, and doctor and patient satisfaction [1,8]. Greater clinical empathy produces better clinical results for both patients and doctors.

A study by Hojat et al. [9] found a significant decline in the empathy scores of medical students upon entry to their clinical years (after the third year). A similar result was found in a study by Chen et al. [10], who found that students' empathy scores decreased at the end of the third year. Kozený et al. [11] reported a different pattern: a constant decline in empathy scores during the first 4 years of medical study, followed by an increase during the following 2 years; however, the authors noted that the average empathy scores of students in their clinical years (years 5-6) were lower compared to students in their preclinical years (years 1-4). On the other hand, regarding stress level, inconsistent pattern were found in previous studies. Studies by Sherina et al. [12] and Melaku et al. [13] found the decline of stress level throughout preclinical years, followed by increase of stress level upon entering clinical year. Other studies showed various pattern of stress level across education year among medical students [14-16].

Previous studies on the correlation between empathy and stress have shown that higher level of distress leads to empathy decline $-a$ trend that is also observable among medical students and tends to be consistent even after graduation [17,18]. As such, it is important to identify how medical students sustain distress that might contribute to empathy decline throughout their education. A study of medical students' empathy by Park et al. [18] found that the empathy score of Korean medical students were lower than their counterparts in Western countries. Variation in people's sentiment and empathy can be influenced by Asian culture including in the less dependence on non-verbal communication and assertiveness $[19,20]$. This also applies to stress and coping mechanisms when interacting with the environment; students from East Asian origin tend to have internally targeted control strategies (e.g., using self-control, waiting, accepting situation, and responsibility), whereas those from Western backgrounds rely more on selfenhancing interpretive control (e.g., the use of positive reappraisal combined with control of external factors or environment) [21].

To increase the understanding of this topic, the present study recruited a sample of undergraduate medical students in one of medical schools in Indonesia to assess the association between their stress and empathy levels.

\section{Methods}

\section{Context}

The undergraduate medical program of the Faculty of Medicine Universitas Indonesia (FMUI) has been im- 
plementing a competency-based curriculum. The school selects students from the high school graduates and the curriculum spans for 5.5 years (3.5-year preclinical and 2-year preclinical course). Some dedicated courses have been implemented to develop students' empathy from year 1 to 3 . The courses encourage students to discuss basic concepts on empathy, explore relevant movies or books, and understand their own perceptions on certain issues and others'. The students also observe medical doctors working with patients in various settings, communicate, and have immersion activities in groups with specific characteristics and needs (children with disability, family of human immunodeficiency virus infection and acquired immune deficiency syndrome patient, etc.) and finally, students reflect on the completed activities and their empathy development. In addition to those experiential-based activities, students usually learn in classroom during preclinical year. During the clinical year which starts in the second semester of year 4 , the teaching and learning activities are more embedded in the daily clinical rotations. Students have numerous patient encounters under supervision, and empathy as well as doctor-patient communications are among competencies to develop at this stage.

\section{Study design}

This study used a cross-sectional design. From February to April 2017, questionnaires measuring student stress and empathy levels were administered to undergraduate medical students in all educational years at FMUI. This study was approved by the Research Ethical Committee in the FMUI (No. 302/UN2.F1.D1/KBK/ PDP.01/2017). Informed consent was obtained from all individual participants included in the study.

\section{Subjects}

The participants were selected from the FMUI database of medical students. The total of matriculating students were 1,198 students, then were categorized into six groups based on their education year and gender. The minimum sample was set at 423 students, yet a total of 464 participated voluntarily in this research. All respondents were undergraduate medical students with active enrolment in FMUI, including both preclinical and clinical-year students.

\section{Instruments}

To measure stress level, this study used the 10-item version of the Perceived Stress Scale (PSS-10), which uses a 4-point Likert scale to score each item. The PSS-10 was translated from English to Indonesian, then back-translated to ensure consistency. In previous studies, the PSS-10 was found to have good reliability, showing a Cronbach's $\alpha$ coefficient above 0.70 , even up to 0.91 [22]. The PSS-10 measures general stress level, so it is not limited to certain situations or settings [23]. To measure empathy level, this study used the Jefferson Scale of Physician Empathy (JSPE) for medical student, which was also translated from English to Indonesian. This instrument was developed by Hojat et al. [24] and was designed to measure empathy level among medical practitioners, showing an internal reliability of 0.87 among physicians and 0.89 among medical students in the original research. In research where the JSPE was translated into Czech and Japanese languages, the Cronbach's $\alpha$ coefficients were also good $(0.70-0.90)$ $[11,20,24]$.

\section{Procedures}

The PSS-10 and the JSPE were translated into Indonesian by the first author. The translated version was then reviewed by the second author. This version was then back-translated by an independent contributor: a physician from Universitas Indonesia who is fluent in 
both languages. The final Indonesian versions of the questionnaires were subsequently compared to the original versions. The translated versions of both questionnaires were considered comparable to the originals [25].

During May and June 2017, the questionnaires were distributed online via Google Forms to FMUI medical students in years $1-5$ of their studies. The authors followed up with each participant to confirm their participation in the study. Those who declined to participate or did not respond were excluded.

\section{Statistical analysis}

The IBM SPSS Statistics ver. 20.0 software package (IBM Corp., Armonk, USA) was used for analysis. The Pearson correlation coefficients were calculated to examine the item-total score correlations. The Cronbach's $\alpha$ coefficients were also calculated to confirm the reliability of the questionnaires. The data distribution was examined using Kolmogorov-Smirnov test and showed abnormal distribution for PSS data $(p<0.05)$ and normal distribution for JSPE data $(p>0.05)$. Therefore, to examine the stress score differences between students of different educational years, the authors used the non-parametric analysis: KruskalWallis test to compare median across educational years, and Mann-Whitney U-test as post-hoc analysis. Given the abnormal data distribution, the stress levels of male and female respondents were also compared using the Mann-Whitney U-test. On the other hand, given the normal data distribution of empathy scores, analysis of variance (ANOVA) test and appropriate post-hoc analysis were used to examine the mean difference among educational years systematically. Finally, to analyse the correlation between stress level, empathy level, and educational year, the authors calculated the Spearman correlation coefficient.

\section{Results}

\section{Subjects}

Of the 464 respondents, there were 192 males (41.38\%) and 272 females (58.62\%). Regarding educational years, there were 103 students in their first year, 101 in their second year, 101 in their third year, 87 in their first clinical year, and 72 in their second clinical year. The proportions of each gender differed across educational years, as the number of respondents were adjusted based on the gender proportion of students in each educational year.

\section{Instrument reliability}

In the PSS-10, the item-total correlation coefficients ranged from 0.396 (item number 7) to 0.790 (item number 2), as presented in Table 1. In the JSPE

\begin{tabular}{cccc}
\hline Table 1. Item-total correlation in the Perceived Stress Scale $(n=464)$ & & \\
\hline No. & Item & Item-total correlation & Significance \\
\hline 1 & $\begin{array}{l}\text { In the last month, how often have you been upset because of something that happened } \\
\text { unexpectedly? }\end{array}$ & 0.748 & 0.000 \\
2 & In the last month, how often have you felt that you were unable to control the important & 0.790 & 0.000 \\
& things in your life? & 0.754 & 0.000 \\
3 & In the last month, how often have you felt nervous and 'stressed'? & 0.000 \\
$4^{\text {al }} \quad$ In the last month, how often have you felt confident about your ability to handle your & 0.539 & \\
$\quad$ personal problems? & & \\
\hline
\end{tabular}




\begin{tabular}{cccc}
\hline Table 1. (Continued) & & \\
\hline No. & Item & Item-total correlation & Significance \\
\hline $5^{\text {al }}$ & In the last month, how often have you felt that things were going your way? & 0.580 & 0.000 \\
6 & In the last month, how often have you found that you could not cope with all the things & 0.729 & 0.000 \\
$\quad$ that you had to do? & & \\
$7^{\text {al }}$ & In the last month, how often have you been able to control irritations in your life? & 0.396 & 0.000 \\
$8^{\text {al }}$ & In the last month, how often have you felt that you were on top of things? & 0.637 & 0.000 \\
9 & In the last month, how often have you been angered because of things that were outside & 0.617 & 0.000 \\
$\quad \quad$ of your control? & & 0.736 & 0.000 \\
$10 \quad$ In the last month, how often have you felt difficulties were piling up so high that you & & \\
\hline
\end{tabular}

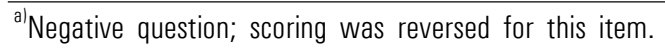

\begin{tabular}{|c|c|c|c|}
\hline No. & Item & Item-total correlation & Significance \\
\hline 1 & $\begin{array}{l}\text { A physician who is able to view things from another person's perspective can render better } \\
\text { care. }\end{array}$ & 0.515 & 0.000 \\
\hline 2 & Physicians' sense of humour contributes to a better clinical outcome. & 0.362 & 0.000 \\
\hline 3 & $\begin{array}{l}\text { Physicians' understanding of their patients' feelings and the feelings of their patients' families } \\
\text { is a positive treatment factor. }\end{array}$ & 0.608 & 0.000 \\
\hline 4 & $\begin{array}{l}\text { For more effective treatment, physicians must be attentive to their patients' personal } \\
\text { experiences. }\end{array}$ & 0.553 & 0.000 \\
\hline 5 & $\begin{array}{l}\text { Understanding body language is as important as verbal communication in physician-patient } \\
\text { relationships. }\end{array}$ & 0.577 & 0.000 \\
\hline 6 & Empathy is an important therapeutic factor in medical treatment. & 0.600 & 0.000 \\
\hline 7 & Patients feel better when their feelings are understood by their physicians. & 0.650 & 0.000 \\
\hline 8 & $\begin{array}{l}\text { Physicians' demonstration of understanding their patients' emotions is an important factor } \\
\text { in interviewing and history taking. }\end{array}$ & 0.636 & 0.000 \\
\hline 9 & $\begin{array}{l}\text { Willingness to imagine oneself in another person's place contributes to providing quality } \\
\text { care. }\end{array}$ & 0.536 & 0.000 \\
\hline $10^{\mathrm{al}}$ & $\begin{array}{l}\text { Patients' illnesses can be cured only by medical treatment; physicians' affectional ties with } \\
\text { their patients do not have a significant place in this endeavour. }\end{array}$ & 0.451 & 0.000 \\
\hline 11 & $\begin{array}{l}\text { Activity in a patient's mind can often be expressed by nonverbal cues, such as facial expressions } \\
\text { or body language, which must be carefully observed by physicians. }\end{array}$ & 0.613 & 0.000 \\
\hline 12 & $\begin{array}{l}\text { A patient who feels understood can experience a sense of validation that is therapeutic } \\
\text { in its own right. }\end{array}$ & 0.696 & 0.000 \\
\hline 13 & $\begin{array}{l}\text { One important component of a successful physician-patient relationship is physicians' abilities } \\
\text { to understand the emotional statuses of their patients and their patients' families. }\end{array}$ & 0.687 & 0.000 \\
\hline 14 & $\begin{array}{l}\text { It is as important to ask patients about what is happening in their lives as it is to ask } \\
\text { about their physical complaints. }\end{array}$ & 0.595 & 0.000 \\
\hline 15 & $\begin{array}{l}\text { It is acceptable for a physician to be touched by intense emotional relationships between } \\
\text { patients and their families. }\end{array}$ & 0.335 & 0.000 \\
\hline 16 & $\begin{array}{l}\text { Reading nonmedical literature and enjoying the arts can enhance physicians' abilities to } \\
\text { render better care. }\end{array}$ & 0.433 & 0.000 \\
\hline $17^{\text {a) }}$ & $\begin{array}{l}\text { Because people are different, it is almost impossible for physicians to see things from their } \\
\text { patients' perspectives. }\end{array}$ & 0.309 & 0.000 \\
\hline $18^{\text {a) }}$ & Emotion has no place in the treatment of medical illness. & 0.488 & 0.000 \\
\hline 19 & Empathy is a therapeutic skill, without which the physician's success will be limited. & 0.577 & 0.000 \\
\hline 20 & The best way to take care of a patient is to think like a patient. & 0.476 & 0.000 \\
\hline
\end{tabular}

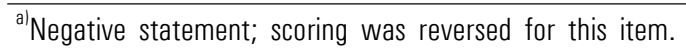


questionnaire, the item-total correlation coefficients ranged from 0.362 (item number 2) to 0.696 (item number 12), as presented in Table 2. All item-total correlation coefficients were statistically significant, which indicates that the direction of the scoring between each item and the total score was positive throughout. The Cronbach's $\alpha$ coefficients were 0.764 for the PSS-10 and 0.731 for the JSPE, which indicates good internal reliability for a psychological study [20].

\section{Stress level across educational years}

Overall, the female students showed higher average stress scores compared to the male students (19.22 versus 17.96, $\mathrm{p}=0.047$ ). Furthermore, when examining the pattern of stress across educational years, it was found that the students' stress levels peaked in the first year and continued to decline in the following years. The Kruskal-Wallis test showed significant variations in stress level between students of different educational years. A post-hoc analysis using the Mann-Whitney test revealed statistically significant differences between first-, second-, and third-year students compared to sixth-year students (second clinical year) (Table 3).

\section{Empathy level across educational years}

The results indicate that female students consistently scored higher than male students in empathy level, regardless of educational year; however, this difference was not statistically significant $(\mathrm{p}=0.246)$. An interesting pattern can also be observed, in which the students' empathy levels increased over the first three preclinical years, declined during the first clinical year, and increased again during the second clinical year. Significant differences between groups were found via hypothetical testing using the ANOVA method. A post-hoc analysis showed significant differences in empathy scores between the third-year and firstclinical-year students ( $\mathrm{p}=0.001)$, as well as between the first- and second-clinical-year students $(\mathrm{p}=0.014)$. The complete results are provided in Table 3.

\section{Correlation between stress and empathy levels}

No significant correlation was found between stress and empathy levels. The Spearman correlation coefficient was -0.031 , with $\mathrm{p}-$ value of 0.502 .

Table 3. The Empathy and Stress Levels at Different Educational Year $(n=464)$

\begin{tabular}{|c|c|c|c|}
\hline Elements & Value & $\mathrm{p}$-value & Post-hoc analysis \\
\hline Stress level (PSS; max score $=40$ ) & & $0.011^{\mathrm{al}}$ & \\
\hline (1) First year & $20(5-36)$ & & $(5)<(1),(2),(3)^{b)}$ \\
\hline (2) Second year & $18(6-40)$ & & \\
\hline (3) Third year & $19(3-34)$ & & \\
\hline (4) First clinical year & $18(5-33)$ & & \\
\hline (5) Second clinical year & $17(1-35)$ & & \\
\hline Empathy level (JSPE; max score $=140$ ) & & $0.003^{\mathrm{cl}}$ & \\
\hline (6) First year & $113.50 \pm 10.036$ & & $(8)>(6),(9)<(7),(8),(10)^{d)}$ \\
\hline (7) Second year & $116.03 \pm 10.922$ & & \\
\hline (8) Third year & $117.49 \pm 10.723$ & & \\
\hline (9) First clinical year & $112.03 \pm 11.251$ & & \\
\hline (10) Second clinical year & $116.21 \pm 9.577$ & & \\
\hline
\end{tabular}

Data are presented as median (min-max) or mean \pm standard deviation.

PSS: Perceived Stress Scale, JSPE: Jefferson Scale of Physician Empathy.

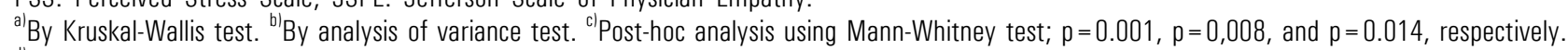

${ }^{d)}$ Post-hoc analysis; $p=0.007, p=0.015, p=0.001$, and $p=0.014$, respectively. 


\section{Discussion}

The present study aims to assess the association between undergraduate medical students' empathy and stress levels. The evaluation of translated instruments showed that the reliability of both questionnaires are satisfactory, which is relatively consistent with previous studies [11,20,22,24]. High internal reliability that does not exceed 0.90 confirms that a questionnaire is valid for its intended use but the items are not redundant [22,24].

Regarding stress level, current study shows that the result is consistent with previous studies whereby higher stress levels were reported among female students compared to male students $[2,14-16,18]$. Females tend to have higher daily stress levels compared to males, and they are more sensitive to the feeling that an event is undesirable [26]. In the present study, the data revealed a stress pattern that peaked during the first year and continued to decline in the following years, reaching its lowest in the final year. These findings are relatively similar to those of a study conducted in Saudi Arabia and South Korea $[14,18]$. In other studies, however, different patterns were found. Another study in Saudi Arabia found the highest prevalence of stress among secondyear students, followed by first-year students [15]. In contrast, a study in Ethiopia reported that stress levels declined during the first 3 years of medical education, increased during the fourth year, and declined once again over the next 2 years [13]. A study in Malaysia showed a similar pattern, but the results were not statistically significant [12].

The highest stress levels, which were found among first-year students, could be related to adaptation process with a new experience on campus life. This period could be challenging to students as they have to adjust to self-directed learning style in university, which is very different from what they experienced in highschool. First-year medical students are young people aged 17-19 years old who are in transition period from childhood to adulthood who are going through complex psychosocial, neuro-biological, and brain structure changes [27]. Brain development during this period is incomplete, which showed in the incongruity between maturation of the limbic (i.e., emotional, reward system) and pre-frontal cortex (i.e., executive function such as decision making); this may explain the high risk for having emotional distress [28]. Besides those risk factors, during data collection, the first-year students were studying biomedical subjects in more depth, which can be challenging and may lead to emotional distress [18]. Likewise, the decline in stress levels that occur over the following years may be influenced by the students' adjustment and their increasing maturity [14,18,28].

Furthermore, higher empathy levels were consistently found among female students compared to male students, regardless of educational year; however, based on the t-test results, this difference between genders was not statistically significant. Nevertheless, it should be noted that similar results were found in previous studies [9-11,20,29]. Other studies found that female doctors tend to be more sensitive to emotional signs and signals expressed by the patient, both verbal and nonverbally, than male doctors [7], and female are also better than male at managing the emotional aspect of empathy, even though they performed about the same in the cognitive aspect of empathy [29,30]. Empathy level measurement which focus on the cognitive aspect might not be able to address the difference between male and female which is probably anchored in the affective aspect of empathy.

The pattern of empathy changed across educational years, which is relatively consistent with previous studies $[9,10]$. A sudden decline in empathy occurred among students in the first clinical year (or fourth year 
of study). These students begin to work in clinical settings with real physician-patient interactions, rather than studying in a classroom as in previous years [31]. Significant differences in mean scores were found between the preclinical students (years 1-3) and the first-clinical-year students, as well as between the firstand second-clinical-year students (Table 3). This pattern is similar with the patterns reported in two previous studies, which showed a decline in empathy upon entering clinical rotation [9-11]. Those studies showed that the decline might be associated with many factors, such as a lack of good role models in the clinical practice, de-idealization of clinical practice experienced by the students, time issues, academic pressure, re-adaptation and even interacting with patients [9-11]. A greater decline can be observed among males (-6.78) than females $(-4.57)$ during the third year, compared to the trends of the first clinical year. This finding is consistent with research by Hojat et al. [9], who found that men are more prone to empathy decline.

However, this study showed that the empathy level bounced back to a higher level in the second clinical year, the pattern which could also be found in several previous studies, especially those from Far East region [32]. This phenomenon could be attributed to decreased burnout level among medical students at this stage as they were more adapted to clinical rotations. In the second clinical year, medical students in FMUI were also entering minor rotations which were considered as less stressful compared to the major rotations (such as internal medicine, obstetric and gynecology, surgery, and pediatrics) during the first clinical year. Also, students' self-reflection on their experiences in interacting with patients at the end of each clinical rotation in the first clinical year might also contribute to the "bounce-back" of the empathy level. In addition to the more specific contextual factors, the "bounce-back" of empathy level in the present study which used Indonesian translated version of JSPE might also be due to the collectivist culture $[32,33]$ in Indonesia and tendency of the students to operate internally targeted control strategies in which they might motivate themselves to take responsibility as future physician dealing with patients and adapt with the expectation of the clinical learning environment [21].

The total score of empathy of medical students in the present setting is comparable to those from previous studies $[9,24]$ and is higher compared to the total scores of Korean medical students [18]. This can probably be attributed to the dedicated courses in the medical curriculum which aim to develop students' empathy and professionalism across the years. In addition, Indonesia is a multi-ethnic country with hierarchical and collectivist culture [33]. While empathy is not merely cognitive but also affective and behavioural capability [8], the influence of culture in empathy is complex and not limited to the non-verbal communication and emotional expression [18]. The influence of hierarchical culture might also exist when students are given chance to observe their senior doctors when dealing with patients. In this regards, positive role-modeling process in showing empathy from the superior level can become a strength point for medical students' empathy development. Studies on the influence of hierarchical culture on feedback [34] and clinical reasoning teaching and learning [35] show that acknowledgement of culture in a certain setting is critical to facilitate development process of medical students' competencies.

The present authors found no significant correlation between level of stress and empathy; however, previous studies reported that distress and burnout are major factors in empathy decline among medical students $[3,29]$. As there are many components of psychological distress, it seems that various underlying factors might be causing empathy decline, rather than general stress 
$[3,29,36]$. Although burnout was found to be a strong factor, depersonalization and depression were not [29]. The explanation provided was that burnout is related to the professional aspect of medicine, while depression is more related to general distress [29], hence in relation to empathy, the measurement of burnout rather than general distress might be more appropriate. The present authors identified this as one of the development for future study. In a systematic review that considered distress as the main factor of empathy decline among medical students, especially those who are just entering clinical rotation, several components of distress were said to produce such a decline, including burnout, depression, reduced quality of life, and low sense of well-being [3].

Despite non-association of general stress and cognitive empathy in this study, the authors would like to highlight that stressors can be interpreted and tackled differently by people coming from different culture [21,37], and it is also the case for medical students. In addition, a longitudinal study on the impact of stress management course for first year medical students also shows that intervention towards medical students without changing the learning environment may not be effective [38]. Therefore, as this study highlights, focusing on preventing students' stress only might not be sufficient to support empathy development in undergraduate medical program. Furthermore, given the diversity of culture in Indonesia, approach to support students well-being and empathy development should incorporate this awareness and attempts to create supportive learning environment.

There are some limitations in the present research. First, this is a cross-sectional study which identify the stress and empathy level at a point of time, in group of medical students from different years of education. Cohort study might be useful to assess the changing of stress and empathy level of the subjects over a period of time in medical training $[20,25]$. Second, this study was conducted in one medical school in Indonesia, hence the generalisation of the results can be limited. However, other medical schools with comparable cultural and curricular settings may find the findings of this study beneficial.

In future studies, other measurements should be applied to assess the various aspects of stress. While many previous studies indicated distress as the main factor of empathy decline, the present study contradicts this finding, at least in the current setting. Further investigations could elaborate on the components of stress and how other relevant variables might affect empathy decline among medical students.

This study examined the empathy and stress levels of FMUI medical students in different educational years, finding no correlation between these variables. While general stress level appeared to decline over time, the authors could hypothesize that the students were better able to manage their stress levels as they matured and adapted to the programme. It is important to note, however, that a sharp decrease in empathy decline can be observed among students entering the first clinical year. Medical educators should explore why such a decline occurred and how to deal with it in the future. The bounced-back of empathy level to a higher level in the second year highlights the importance of student adaptation in the clinical learning environment and the support system. It is undeniable that clinical empathy contributes to better patient-oriented care, as well as to patient and physician satisfaction; therefore, further research is required to explore the other underlying factors of empathy decline among medical students. 


\section{ORCID:}

James Wiguna Wahjudi: https://orcid.org/0000-0001-8908-1131;

Ardi Findyartini: https://orcid.org/0000-0002-9601-3994;

Fransiska Kaligis: https://orcid.org/0000-0003-3776-7064

Acknowledgements: We would like to thank all undergraduate medical students in FMUI who participated voluntarily in this study. We would also like to extend our gratitude to Dr Aria Kekalih who provided feedback on the statistical analysis.

Funding: We would like to acknowledge the support of Faculty of Medicine, Universitas Indonesia for providing the publication grant for the present study.

Conflict of interest: No potential conflict of interest relevant to this article was reported.

Author contributions: Designed and conducted the study, completed data collection and analysis, and contributed in the manuscript development: JW; designed and conducted the study, completed data analysis, and led the manuscript development: AF; completed data analysis and contributed in the manuscript development: FK; and all authors approved the final version of the manuscript.

\section{References}

1. Dyrbye LN, Thomas MR, Shanafelt TD. Medical student distress: causes, consequences, and proposed solutions. Mayo Clin Proc. 2005;80(12):1613-1622.

2. Iqbal S, Gupta S, Venkatarao E. Stress, anxiety and depression among medical undergraduate students and their socio-demographic correlates. Indian J Med Res. 2015;141(3):354-357.

3. Neumann M, Edelhäuser F, Tauschel D, Fischer MR, et al. Empathy decline and its reasons: a systematic review of studies with medical students and residents. Acad Med. 2011;86(8):996-1009.
4. Newton BW. Walking a fine line: is it possible to remain an empathic physician and have a hardened heart? Front Hum Neurosci. 2013;7:233.

5. Roberto KJ. Stress..what stress?: an examination of stress, stress resiliency, and performance adaptation. Ann Arbor, USA: ProQuest Information and Learning Company; 2006.

6. Halpern J. What is clinical empathy? J Gen Intern Med. 2003; 18(8):670-674.

7. Hojat M, Gonnella JS, Nasca TJ, Mangione S, Vergare M, Magee M. Physician empathy: definition, components, measurement, and relationship to gender and specialty. Am J Psychiatry. 2002;159(9):1563-1569.

8. Mercer SW, Reynolds WJ. Empathy and quality of care. Br J Gen Pract. 2002;52 Suppl:S9-S12.

9. Hojat M, Vergare MJ, Maxwell K, et al. The devil is in the third year: a longitudinal study of erosion of empathy in medical school. Acad Med. 2009;84(9):1182-1191.

10. Chen D, Lew R, Hershman W, Orlander J. A crosssectional measurement of medical student empathy. J Gen Intern Med. 2007;22(10):1434-1438.

11. Kozený J, Tisanská L, Höschl C. Assessing empathy among Czech medical students: a cross-sectional study. Cesk Psychol. 2013;57(3):246-254.

12. Sherina MS, Rampal L, Kaneson N. Psychological stress among undergraduate medical students. Med J Malaysia. 2004;59(2):207-211.

13. Melaku L, Mossie A, Negash A. Stress among medical students and its association with substance use and academic performance. J Biomed Educ. 2015;2015: 149509. https://doi.org/10.1155/2015/149509.

14. Abdulghani HM, AlKanhal AA, Mahmoud ES, Ponnamperuma GG, Alfaris EA. Stress and its effects on medical students: a cross-sectional study at a college of medicine in Saudi Arabia. J Health Popul Nutr. 2011; 29(5):516-522.

15. Sani M, Mahfouz MS, Bani I, et al. Prevalence of stress 
among medical students in Jizan University, Kingdom of Saudi Arabia. Gulf Med J. 2012;1(1):19-25.

16. Shah M, Hasan S, Malik S, Sreeramareddy CT. Perceived stress, sources and severity of stress among medical undergraduates in a Pakistani medical school. BMC Med Educ. 2010;10:2.

17. Dahlin M, Joneborg N, Runeson B. Stress and depression among medical students: a cross-sectional study. Med Educ. 2005;39(6):594-604.

18. Park KH, Kim DH, Kim SK, et al. The relationships between empathy, stress and social support among medical students. Int J Med Educ. 2015;6:103-108.

19. Hong M, Lee WH, Park JH, et al. Changes of empathy in medical college and medical school students: 1-year follow up study. BMC Med Educ. 2012;12:122.

20. Kataoka HU, Koide N, Ochi K, Hojat M, Gonnella JS. Measurement of empathy among Japanese medical students: psychometrics and score differences by gender and level of medical education. Acad Med. 2009;84(9):11921197.

21. Tweed RG, White K, Lehman DR. Culture, stress, and coping: internally-and externally-targeted control strategies of European Canadians, East Asian Canadians, and Japanese. J Cross Cult Psychol. 2004;35(6):652-668.

22. Lee EH. Review of the psychometric evidence of the perceived stress scale. Asian Nurs Res (Korean Soc Nurs Sci). 2012;6(4):121-127.

23. Cohen S. Perceived stress in a probability sample of the United States. In: Spacapan S, Oskamp S, eds. The Social Psychology of Health: The Claremont Symposium on Applied Social Psychology. Thousand Oaks, USA: Sage Publications; 1988:31-67.

24. Hojat M, Mangione S, Nasca TJ, et al. The Jefferson Scale of Physician Empathy: development and preliminary psychometric data. Educ Psychol Meas. 2001; 6l(2):349-365.

25. Di Lillo M, Cicchetti A, Lo Scalzo A, Taroni F, Hojat M.
The Jefferson Scale of Physician Empathy: preliminary psychometrics and group comparisons in Italian physicians. Acad Med. 2009;84(9):1 198-1202.

26. Matud MP. Gender differences in stress and coping styles. Per Individ Dif. 2004;37(7):1401-1415.

27. Martel A, Fuchs DC. Transitional age youth and mental illness: influences on young adult outcomes. Child Adolesc Psychiatr Clin N Am. 2017;26(2):13-17.

28. Wilens TE, Rosenbaum JF. Transitional aged youth: a new frontier in child and adolescent psychiatry. J Am Acad Child Adolesc Psychiatry. 2013;52(9):887-890.

29. Paro HB, Silveira PS, Perotta B, et al. Empathy among medical students: is there a relation with quality of life and burnout? PLoS One. 2014;9(4):e94133.

30. Vongas JG, Al Hajj R. The evolution of empathy and women's precarious leadership appointments. Front Psychol. 2015;6:1751.

31. Faculty of Medicine Universitas Indonesia. The curriculum of Faculty of Medicine Universitas Indonesia. Central Jakarta, Indonesia: Faculty of Medicine Universitas Indonesia; 2012.

32. Ponnamperuma G, Yeo SP, Samarasekera DD. Is empathy change in medical school geo-socioculturally influenced? Med Educ. 2019;53(7):655-665.

33. Hofstede G. Culture's consequences: comparing values, behaviors, institutions, and organizations across nations. 2nd ed. Thousand Oaks, USA: Sage Publications; 2001.

34. Suhoyo Y, van Hell EA, Kerdijk W, et al. Influence of feedback characteristics on perceived learning value of feedback in clerkships: does culture matter? BMC Med Educ. 2017;17(1):69.

35. Findyartini A, Hawthorne L, McColl G, Chiavaroli N. How clinical reasoning is taught and learned: cultural perspectives from the University of Melbourne and Universitas Indonesia. BMC Med Educ. 2016;16:185.

36. Thomas MR, Dyrbye LN, Huntington JL, et al. How do distress and well-being relate to medical student 
empathy?: a multicenter study. J Gen Intern Med. 2007; 22(2):177-183.

37. Hashimoto T, Mojaverian T, Kim HS. Culture, interpersonal stress, and psychological distress. J Cross Cult Psychol. 2012;43(4):527-532.
38. Dyrbye LN, Shanafelt TD, Werner L, Sood A, Satele D, Wolanskyj AP. The impact of a required longitudinal stress management and resilience training course for first-year medical students. J Gen Intern Med. 2017; 32(12):1309-1314. 